\title{
Proof of Concept Validation of a Swimming Multi Sensor Platform for In-Situ Ocean Monitoring
}

\author{
Julius Harms, Thorsten A. Kern \\ Institute for Mechatronics in Mechanics (iMEK) \\ Hamburg University of Technology (TUHH), 21073 Hamburg \\ Corresponding author: Julius.harms@tuhh.de
}

\begin{abstract}
Summary:
A concept for a modular multi sensor platform to collect various measurement data at the interface of air and water is presented in this paper. Besides position tracking and wave height measurement, the drifting buoy allows individual integration of different sensors by its scalable design. A wave energy converter and solar modules provide redundant energy harvesting sources for long time applications. The concept was tested in the Baltic Sea off the coast of Fehmarn, by a first prototype equipped with low-cost temperature and salinity sensor. Drifting behaviour was compared to the CARTHE Drifter.
\end{abstract}

Keywords: In-Situ, Monitoring, Drifter, Modular, Platform, Maritime, Ocean

\section{Introduction}

Existing drifter platforms, used to gather ocean current information by tracking the buoy's position, lack the ability to measure individual parameters, have a limited energy source or are too expensive for the use in large quantities. Big experiments like LASER [1], using 1000 drifter at the same time, show that there is a great need for low-cost platforms. The used CARTHE drifter, like others, miss the ability to acquire further measurement data like e.g. temperature or salinity. At the same time, measurement data with high space resolution present a great advantage for many applications like analysation of submesoscale dynamics. The here presented concept is the development of an affordable multi-sensor platform with high modularity in its use.

\section{Concept Description}

The modularity of the concept is given by the system reduction to the most important standardized components, combined with an extension concept for customized components. Figure 1a shows the schematic of the Affordable Multi Sensor Drifter (AMuSeD) with the main unit as the central component. With the integrated GPS-Module and a small MEMS IMU, the position and wave height is always acquired. If further measurement data is required, the embedded master controller requests measurement data from additional sensor unit, replying according to a standardized protocol. This protocol allows exchange of the sensors on the sensor unit without touching the main unit. The main unit processes the received data and transmits the full data set via satellite communication. This modularity allows research groups to use the

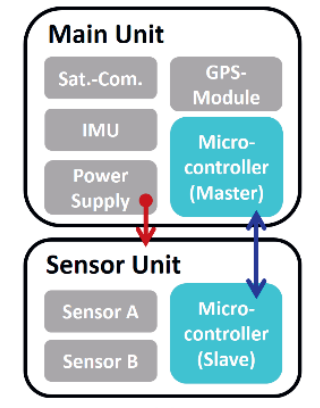

(a)

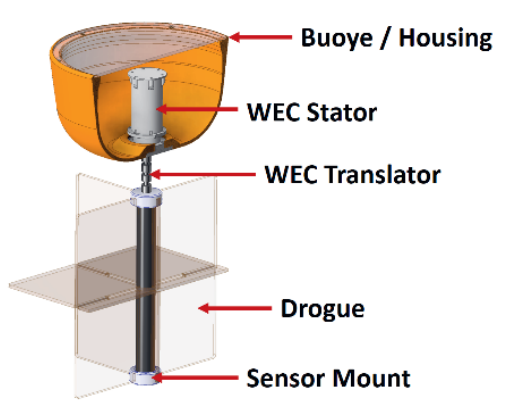

(b)
Figure 1: (a) Schematic for the sensor interfacing (b) of the $\mathrm{AMuSeD}$ prototype

main unit as their key component and adjust only the sensor unit according to their individual needs. Besides the data processing, the main unit provides a power supply for all peripherals, which can be implemented using energy harvesting methods if long time measurement is required. Since solar power, in some areas, is not sufficient, a wave energy converter (WEC) adds redundancy to the supply. The WEC is based on a specially developed linear generator and the planned implementation can be seen in Figure $1 \mathrm{~b}$. The energy is gained from a relative movement between stator and translator, due to the difference in the wave orbital velocity exciting the buoy and drogue. The concept was tested in a wave channel and the generator simulated based on electromagnetic FEM software. The promising results will be presented in the scope of a different work. 


\section{Prototype Test}

In a proof of concept experiment, a prototype of AMuSeD was built and tested. The complexity of the main unit was reduced to electronics for the acquisition of GPS position and acceleration. The connected sensor unit consists of low-cost temperature and conductivity sensors. The temperature sensor was implemented by a PT1000 $(\sim 15 €)$ with the RTD-Digital converter MAX31865 ( 5€). The integrated ADC allows a resolution of $0.03^{\circ} \mathrm{C}$ and a total accuracy over all operating conditions of $0.5^{\circ} \mathrm{C}$. Conductivity was measured by the Atlas Scientific Conductivity K1.0 Kit $(\sim 230 €)$, which gives an accuracy of $+/-2 \%$.

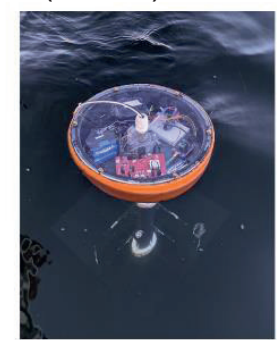

(a)

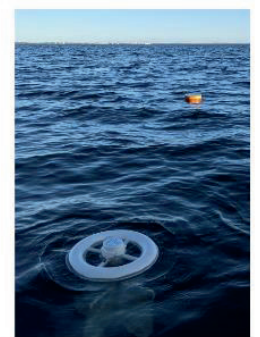

(b)

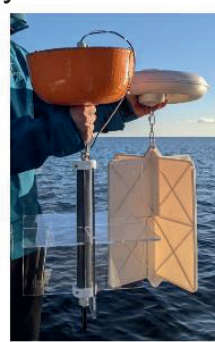

(c)
Figure 2: (a) Top view AMUSeD (b) deployed CARTHE \& AMuSeD (c) comparison AMuSeD (l.) CARTHE (r.)

This 2-electrode conductive sensor allows an inexpensive use, but has the disadvantage of being more susceptible to bio fouling and polarization. For this reason, the authors of this paper are researching on low-cost inductive salinity sensors [2]. Two of the AMuSeD drifters, one with full electronics and one with GPS tracking only, were deployed on the $27^{\text {th }}$ of November 2020 together with two CARTHE drifter off the coast of Fehmarn (Fig. 2b/c). For the duration of 280 minutes, the drifter covered a distance of $\sim 2.5 \mathrm{~km}$ (Fig. 3a) while one was tracking measurement data every minute. After the simultaneous deployment, the measuring platforms drifted close together during the entire period. At the time of collection, the CARTHE
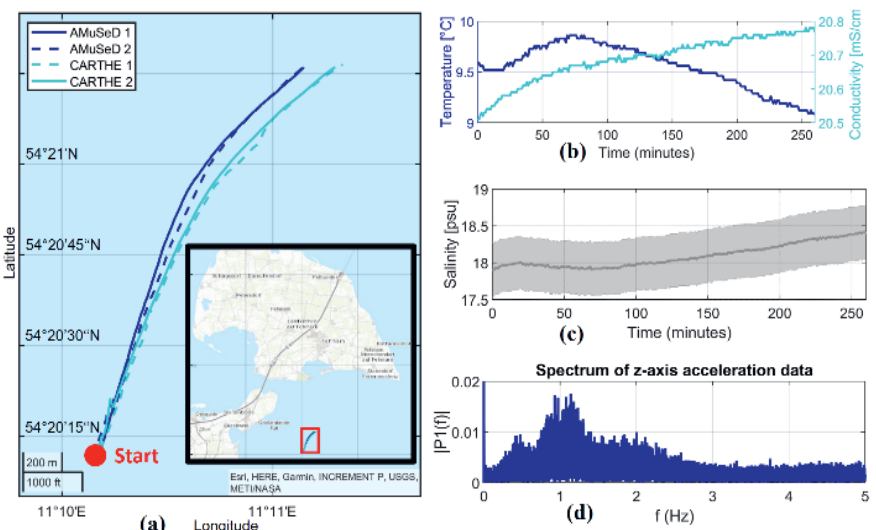

Figure 3: (a) Comparison of GPS-tracks (b) temperature \& conductivity over time (c) calculated salinity with error band (d) frequency spectrum of vertical acceleration data drifters were $150 \mathrm{~m}$ further east than the AMuSeD, which may be due to the slightly different sizes of the drogues and the waves coming from the northwest. The tracked temperature and conductivity data (Fig $3 \mathrm{~b}$ ) is used to calculate the water salinity (Fig 3c) based on the practical salinity scale [3]:

$$
S=a_{0}+a_{1} K^{\frac{1}{2}}+a_{2} K+a_{3} K^{\frac{3}{2}}+a_{4} K^{2}+a_{5} K^{\frac{5}{2}}
$$

Where the salinity $S$ is calculated from the empirical parameters $a_{0}$ to $a_{5}$ and the conductivity ratio $K$ of the sample to a standard $\mathrm{KCl}$-solution at $15^{\circ} \mathrm{C}$. With the given the measurement uncertainties, this leads to an propagated uncertainty of $+/-0.035$ psu $(\sim 2 \%)$ for the present water conditions of around $9.5^{\circ} \mathrm{C}$ temperature and a water conductivity of $20.600 \mu \mathrm{S} / \mathrm{cm}$. A spectral analysis of the acceleration data (Fig $3 \mathrm{c}$ ) gives information about sea state, wave height and period.

\section{Results and Conclusion}

The collected measurement data allow a much better interpretation than a mere observation of the position data. Despite the low-cost sensors, the additional measurement data achieve accuracy values to identify disturbances like changing sea states and temperature jumps that affect the drift behaviour. This is especially relevant for remote long-term measurements. The executed test is a successful proof of concept. In the next steps, the modular electronics will be integrated by a PCB design and the energy supply will be ensured by energy harvesting. In addition, the housing will be reduced in size and optimized for better drift behavior while being easy to produce. The ultimate goal is to use biocompatible materials in the manufacturing process so that even when large quantities are used, the oceans are not polluted. In addition to the main unit development, research on low-cost sensors, especially inductive conductivity sensors, with high resolution is going to be performed.

\section{References}

[1] Novelli et al. "A Biodegradable Surface Drifter for Ocean Sampling on a Massive Scale" . J. Atmos. Oceanic Technol. (2017) 34, 2509-2532; doi:10.1175/JTECH-D-17-0055.1

[2] Harms et al. "Theory and Modelling of eddy current type Inductive Conductivity Sensors" MDPI Eng. Proc. (2021) (accepted)

[3] E. Lewis, "The practical salinity scale 1978 and its antecedents" IEEE Journal of Oceanic Engineering, vol. 5, no. 1, pp. 3-8, January 1980, doi:10.1109/JOE.1980.1145448. 